\title{
High-efficiency single-mode Raman generation in a liquid-filled photonic bandgap fiber
}

\author{
Sylvie Lebrun, Philippe Delaye, Robert Frey, and Gérald Roosen \\ Laboratoire Charles Fabry de l'Institut d'Optique, CNRS, Université Paris-Sud, Campus Polytechnique, RD128, 91127 \\ Palaiseau cedex, France
}

Received September 13, 2006; revised November 13, 2006; accepted November 13, 2006; posted November 16, 2006 (Doc. ID 75031); published January 26, 2007

\begin{abstract}
Single-spatial-mode Raman generation in an ethanol-filled photonic bandgap fiber is demonstrated. Due to the limited bandwidth of the fiber, the generation is limited to the first Stokes order only, allowing high generated power without any visible decrease of the conversion efficiency. The realization of these two key properties opens the way to the realization of optimized compact nonlinear wavelength converters that will accommodate a large variety of usable liquids. (C) 2007 Optical Society of America

OCIS codes: $190.5650,060.2320,060.4370$.
\end{abstract}

Since their appearance one decade ago, photonic crystal fibers have led to numerous developments in various domains of fiber technologies. ${ }^{1-3}$ This is especially true in the field of nonlinear optics, where the possibility of decreasing the mode field diameter and tailoring the dispersion of the fiber has opened the way to the optimization of numerous nonlinear devices, such as supercontinuum sources ${ }^{4}$ or parametric sources of correlated photons. ${ }^{5}$ Despite the great advances brought by these fibers, they are still limited by the use of silica as nonlinear material. The use of photonic crystal fiber with a core material with higher nonlinear susceptibility such as liquid or gases $^{6-9}$ would lead to even higher increases in the performance of nonlinear devices and new applications brought by the large variety of usable liquids and gases. Besides, liquid- and gas-filled photonic crystal fibers have several original propagation properties, including transmission bands that can be used to favor or prevent a given nonlinear mechanism. In the case of the stimulated Raman scattering experiments described here, the Raman cascade can be stopped to a given Stokes order by placing the next order outside the transmission band. The chosen Stokes order is then generated efficiently with high power output without being depleted by the nextorder generation.

The use of gases with an index very close to 1 does not drastically change the propagation properties of hollow-core photonic crystal fibers, ${ }^{6,7}$ but this is not the case with liquids, which usually have higher refractive indices. Until now nonlinear experiments with liquid-core photonic crystal fibers ${ }^{8}$ have used an air-filled cladding, requiring that the cladding holes at both extremities of the fiber be closed. Despite interesting results, this first experiment with this approach suffered from several drawbacks. The first is the multimode structure of the generated beam, which limits its practical use as a Raman generator. The second is the generation of multiple Stokes orders that limit the power transferred to the first Stokes order. In this Letter we present what is, to the best of our knowledge, the first implementation of a single-mode single Raman order generator using a totally liquid-filled photonic bandgap fiber, opening the way toward high-efficiency single-mode Raman sources.

The fiber that is the basis of our experiment is a commercial hollow-core photonic bandgap fiber. The choice of the fiber was governed by several constraints mainly linked to the choice of the liquid and of the pump beam used in our nonlinear experiment. Our aim was to observe Raman generation in ethanol pumped by a frequency-doubled Nd:YAG laser, so the filled fiber should have good transmission for both the pump at $532 \mathrm{~nm}$ and the first Stokes order of ethanol at $630 \mathrm{~nm}$ (Raman shift of $2928 \mathrm{~cm}^{-1}$ ). To take into account the shift of the transmission band ${ }^{10}$ due to filling with a liquid of refractive index 1.36, we had to choose a hollow-core photonic bandgap fiber with a band located in the $1 \mu \mathrm{m}$ range when used with air. Our final choice was HC-1060-02 hollow-core photonic bandgap fiber from CrystalFiber, ${ }^{11}$ which presents a band larger than $100 \mathrm{~nm}$ centered at $1064 \mathrm{~nm}$. The fiber is fixed at both extremities in two tanks closed by a glass window. The fiber is filled from one end using capillary forces, waiting just the necessary time ${ }^{12}$ to fill all the smaller cladding holes, then the second tank is filled with ethanol. Once filled with ethanol, the measured transmission band of the fiber is shifted to $580 \mathrm{~nm}$ with a $3 \mathrm{~dB}$ bandwidth that extends from about 450 to $670 \mathrm{~nm}$. One important point is that, if the pump and the first Stokes wavelength lie in the band as expected, the second Stokes order of ethanol potentially emitted at $772 \mathrm{~nm}$ lies out of the propagation band of the fiber and will then not be generated.

The experimental setup for the characterization of the fiber and of stimulated Raman scattering is identical to the one used in our previous experiment, ${ }^{8}$ simply adding at the output an imaging system to observe the near field of the fiber. The laser was a frequency-doubled nanosecond Nd:YAG microchip laser delivering $0.56 \mathrm{~ns}$ pulses at $532 \mathrm{~nm}$ at a $6 \mathrm{kHz}$ repetition rate and energies up to $1.26 \mu \mathrm{J}$. The near field observed at the exit of the fiber was first measured with both green and red filters [Fig. 1(a) and 1(b)], using a large-area white-light source exciting all the modes of the fiber. The transmitted modes are nearly circular at both wavelength ranges and show 


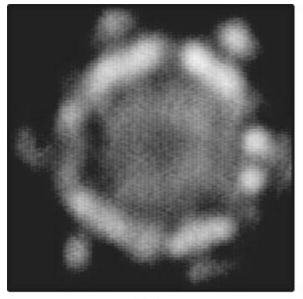

(a)

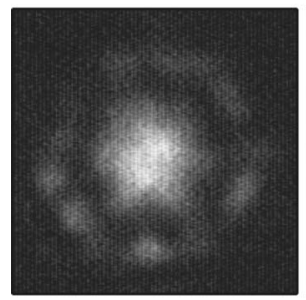

(c)

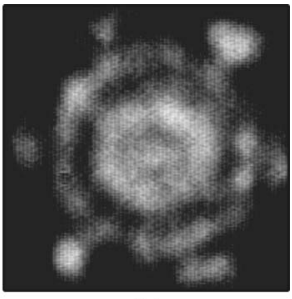

(b)

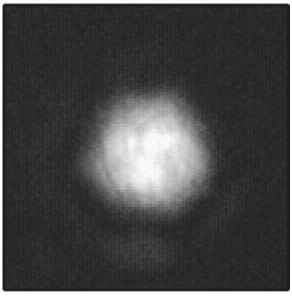

(d)
Fig. 1. Near field of the fiber output obtained with a white-light source using (a) a green filter and (b) a red filter. Also shown are the near field of (c) the pump beam and (d) the first Stokes order beam. The petal-shaped structures around the Gaussian core are due to the periodic structure of the cladding.

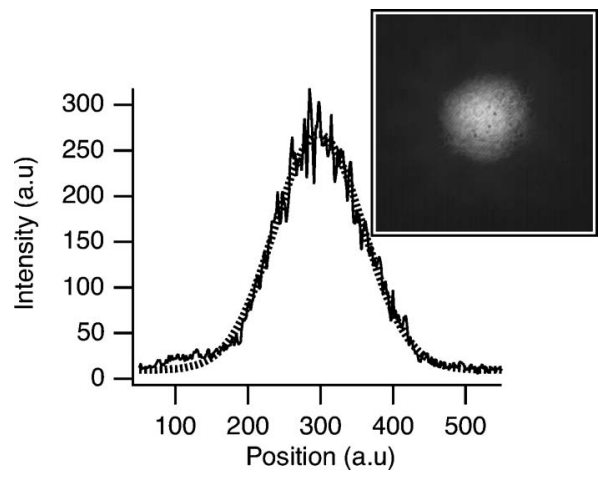

Fig. 2. Intensity profile of the Stokes beam far field (shown in the inset). The dotted curve shows a Gaussian adjustment of the profile.

multimode structures with the presence of petal structures that are classically observed in this kind of fiber ${ }^{10,11}$ and are due to the periodic structures of the cladding. By use of the pump laser, the near field was also observed at both the pump and Stokes wavelengths [Figs. 1(c) and 1(d)]. The pump beam's modal structure is rather similar to the one observed with the white-light source with a closer to Gaussian mode structure, which is essentially due to better coupling of the laser to the core modes. On the other hand, the modal structure at the Stokes wavelength shows better quality and emits a single quasiGaussian mode with very low power in the petal lobes. These lobes can be considered part of the mode spatial structure that reflects the cladding symmetry, ${ }^{3,11}$ which is no longer cylindrical as in usual silica fibers. This single-mode operation is also encountered in the far field, where a nearly Gaussian profile is measured (Fig. 2). This better quality is due to the nonlinear origin of the Stokes line, which favors the Gaussian part of the pump mode that has a higher power density and thus sees higher Raman gain. The mode full width at half-maximum is $4 \mu \mathrm{m}$ at both wavelengths, smaller than the fiber core diameter, which is $9.7 \mu \mathrm{m}$.

A typical measurement of the transmission of the fiber as a function of the incident pump power is shown in Fig. 3. We observe a linear transmission of the pump beam, with a slope of $51 \%$, until the threshold for the appearance of the first Stokes order around $450 \mathrm{~W}$ (corresponding to a pulse energy of $250 \mathrm{~nJ})$. Then the transmitted pump beam intensity saturates, whereas the first Stokes order intensity increases with a double slope behavior. Below $1200 \mathrm{~W}$, we measure a slope of $31 \%$ in the regime of saturation of the pump beam and above $1200 \mathrm{~W}$, a slope of $13 \%$ when the conversion efficiency regime is reached. We note that no saturation of the conversion is seen even at the pump intensities five times above the threshold that we were able to reach, contrary to previous measurements, ${ }^{8}$ where the second Stokes order appears at power only two times above the first-order threshold. In the present case, as the second Stokes order wavelength (at $772 \mathrm{~nm}$ ) lies outside the transmission band of the fiber, it is not guided and thus sees very high losses and is prevented from being generated. The measured intensities are in good qualitative agreement with the theoretical curves $^{13,8}$ for the part where the second Stokes is prevented from appearing. The main difference is due to the slightly multimode structure of the pump beam, which leads to a bad overlap of these higher-order spatial modes of the pump with the single-mode Stokes beam. The energy of the pump beam is only partially coupled to the Stokes beam and is thus not completely depleted at high power, as predicted by the single-mode model. ${ }^{13}$ This bad coupling is probably also responsible for the greater extension of the regime of saturation of the pump beam (between 450 and $1200 \mathrm{~W}$ ). Above $1200 \mathrm{~W}$ the pump beam power continues to slowly decrease as some of the energy of the higher-order spatial modes is finally coupled to the Gaussian Stokes mode.

Comparing the slope of the transmitted first Stokes order intensity (13\%) in the conversion efficiency regime (above $1200 \mathrm{~W}$ incident power) with the transmission of the pump beam below threshold (51\%), we obtain a value of $25 \%$ for the intensity Raman conversion (i.e., a 30\% photon number Raman conversion). This rather good result is probably limited by the relatively high losses of ethanol at $630 \mathrm{~nm}$ as well

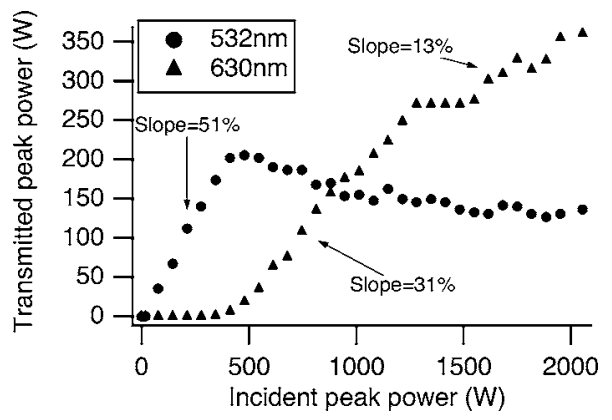

Fig. 3. Plot of the peak intensity transmitted by the fiber at the pump (circles) and the first Stokes order (triangles) wavelengths as a function of the incident peak power. 


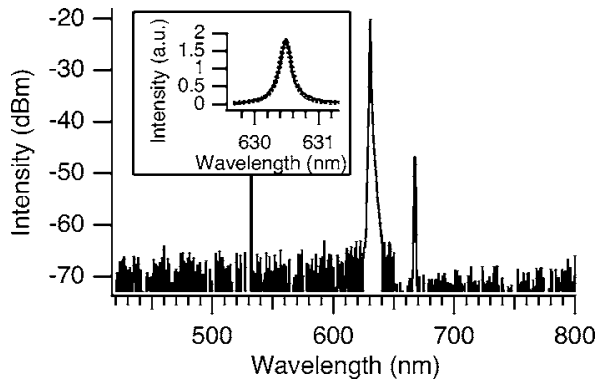

Fig. 4. Spectra transmitted through the ethanol-filled photonic bandgap fiber at the highest pump power. The inset shows the Lorentzian shape with a width of $0.21 \mathrm{~nm}$ FWHM (dashed curve fit) of the first Stokes order.

as the lower modal transmission of the fiber at this wavelength, which is closer to the transmission band edge. This would mean that the $1.14 \mathrm{~m}$ long fiber used is certainly not optimal and that a shorter fiber would certainly give a better conversion efficiency not limited by the losses. Beyond this demonstration an optimization of the fiber for operation with a liquid will probably be necessary to improve the performance.

To confirm the absence of the second Stokes order that is necessary to obtain very high intensities at the first Stokes order, we send the output beam into an optical spectrum analyzer. We could see only the pump and Stokes lines, which clearly shows the advantage of the band control provided by the photonic bandgap fiber. At only the highest pump power, a third line could be seen to appear at $667 \mathrm{~nm}$ (Fig. 4). This very low intensity line $(30 \mathrm{~dB}$ below the Stokes line of interest) has been identified as the $883 \mathrm{~cm}^{-1}$ Raman line of ethanol ${ }^{14}$ pumped by the beam at $630 \mathrm{~nm}$. As the main Raman line of ethanol at $2928 \mathrm{~cm}^{-1}$ is prevented from being emitted, this second highest Raman line of ethanol (usually blocked by main line emission) that lies in the fiber band, is then authorized to be generated. This phenomenon, already observed in hydrogen-filled fibers, ${ }^{7}$ where it allows observation of the rotational lines, will have to be taken into account to set the band of the fiber correctly (for example, by adjusting the index of the liquid by using a mixture of liquids) and to prevent the appearance of all the Stokes lines, which may cause depletion of the first Stokes order.

Beyond the control of the appearance of the higher Stokes order demonstrated here, the use of gas- or liquid-filled photonic bandgap fiber transmission bands can be extended to other nonlinear mechanisms. We can, for example, use the large Stokes shift of liquids and gases to place the Raman line outside the transmission band, keeping the pump power available for the generation of other nonlinear effects such as parametric generation. Such an architecture could be very promising for the generation of correlated photons, where the main limitation comes from the uncorrelated Raman photons generated simultaneously with the correlated photons. ${ }^{5}$ Besides, identically to silica core photonic crystal fibers, the dispersion of liquid- or gas-filled fibers can be optimized to favor phase-matched nonlinear effect such as Stokes-anti-Stokes coupling in stimulated Raman scattering or parametric amplification through fourwave mixing. Another advantage of the use of a more efficient nonlinear core material is to relax the constraint on the core diameter, leading to fibers with a mode field diameter compatible with usual singlemode fibers, allowing better and easier coupling of these new fibers with usual fiber components.

We have realized and characterized a highefficiency single-spatial-mode Raman generator that uses an ethanol-filled photonic bandgap fiber. Despite the nonoptimized nature of the commercial fiber used, good performance was obtained, and the technique presents good opportunities for the realization of Raman amplifiers and generators that are able to operate in the $\mathrm{CW}$ regime using optimized fibers. This architecture combining the nonlinear efficiency of liquids with the low propagation losses of fibers opens the way to a new generation of nonlinear optical devices with high efficiency and tailored properties.

P. Delaye's e-mail address is philippe.delaye @institutoptique.fr.

\section{References}

1. A. M. Zheltikov, Phys. Usp. 43, 1125 (2000).

2. P. St. J. Russell, Science 299, 358 (2003).

3. A. Bjarklev, J. Broeng, and A. Sanchez Bjarklev, Photonic Crystal Fibres (Kluwer, 2003).

4. J. M. Dudley, L. Provino, N. Grossard, H. Maillotte, R. S. Windeler, B. J. Eggleton, and S. Coen, J. Opt. Soc. Am. B 19, 765 (2002).

5. J. G. Rarity, J. Fulconis, J. Duligall, W. J. Wadsworth, and P. St. J. Russell, Opt. Express 13, 534 (2005).

6. F. Benabid, J. C. Knight, G. Antonopoulos, and P. St. J. Russell, Science 298, 399 (2002).

7. F. Benabid, G. Bouwmans, J. C. Knight, P. St. J. Russell, and F. Couny, Phys. Rev. Lett. 93, 123903 (2004).

8. S. Yiou, P. Delaye, A. Rouvie, J. Chinaud, R. Frey, G. Roosen, P. Viale, S. Février, P. Roy, J. L. Auguste, and J. M. Blondy, Opt. Express 13, 4786 (2005).

9. R. Zhang, J. Teipel, and H. Giessen, Opt. Express 14, 6800 (2006).

10. G. Antonopoulos, F. Benabid, T. A. Birks, D. M. Bird, J. C. Knight, and P. St. J. Russell, Opt. Express 14, 3000 (2006).

11. http://www.crystal-fibre.com/datasheets/HC-106002.pdf

12. K. Nielsen, D. Noordegraaf, T. Sørensen1, A. Bjarklev, and T. P. Hansen, J. Opt. A, Pure Appl. Opt. 7, L13 (2005).

13. R. H. Stolen, C. Lee, and R. K. Jain, J. Opt. Soc. Am. B 1, 652 (1984).

14. R. R. Alfano and S. L. Shapiro, Phys. Rev. Lett. 29, 1655 (1972). 